\title{
Lyophilized Black Raspberry Lozenge
}

National Cancer Institute

\section{Source}

National Cancer Institute. Lyophilized Black Raspberry Lozenge. NCI Thesaurus. Code C99163.

A lozenge containing lyophilized black raspberry with potential antioxidant, pro-apoptotic, anti-ang iog enic and chemopreventive activities. In addition to vitamins, minerals and phytosterols, black raspberries are rich in phenolic acids, such as gallic acid, ellagic acid, proanthocyanidins, and flavonoids. The anthocyanins appear to contribute significantly to this agent's effects. Anthocyanins inhibit both the activation of several signal transduction pathways, including the mitogen-activated protein kinase-mediated pathways, and certain transcription factors, such as nuclear factor kappa B (NF-kB), activator protein-1 (AP-1) complex, and nuclear factor in activated T-cells (NFAT). This inhibitory activity modulates the expression of downstream target genes that are upregulated in a variety of cancer cell types, including inducible nitric oxide synthase, cyclooxygenase-2, vascular endothelial growth factor and the anti-apoptotic protein survivin. 